\title{
EDITORIAL
}

\section{A novel preclinical model for rheumatoid arthritis research}

\author{
Frank Petersen and Xinhua Yu* \\ See related research by Vierboom et al., http://arthritis-research.com/content/12/5/R200
}

\begin{abstract}
Based on increasing knowledge on the pathogenesis of rheumatoid arthritis (RA), more and more potential therapeutics have been developed. To evaluate their therapeutic efficacy, safety and toxicity, appropriate animal models are required. Although rodent models of RA have been extensively used for preclinical evaluation, the differences between rodents and humans limit their usability for some species-specific therapeutics. Therefore, autoimmune arthritis developed in a non-human primate with essential hallmarks of RA will be an alternative model for preclinical studies.
\end{abstract}

In the previous issue, Vierboom and colleagues [1] report on a novel animal model of rheumatoid arthritis (RA) induced in common marmosets by immunization with bovine/chicken type II collagen (CII). As a cartilagespecific protein, CII is constitutively expressed in the synovial joint, which is the tissue predominantly affected in RA. CII has been suspected to be an important autoantigen and was used to establish several animal models of RA. In 1977, the first collagen-induced arthritis (CIA) model was established in rats by immunization with CII [2]. Subsequently, this CIA model was reproduced in mouse and monkey in 1980 and 1986, respectively [3,4]. The essential hallmarks of CIA - for example autoimmune response-mediated synovitis and subsequent erosion in cartilage and bone - resemble RA, which makes it the most widely used animal model for RA research.

The rodent model of CIA has been extensively used for investigation of the pathogenesis of the disease and the identification of novel therapeutics and for preclinical studies in RA research. Due to a large evolutionary

\footnotetext{
*Correspondence: xinhuayu@fz-borstel.de

Department of Immunology and Cell Biology, Research Center Borstel, Parkallee 22, D-23845 Borstel, Germany
}

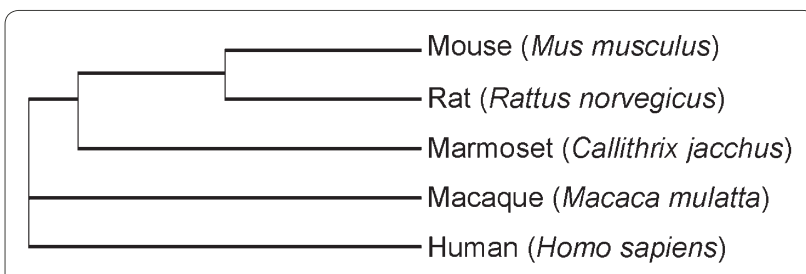

Figure 1. Phylogenetic tree of animal species used in CIA. The phylogenetic tree was generated based on mitochondrial DNA sequences (whole mtDNA except the D-loop region) of the species indicated using ClustalW2 software [13].

distance (Figure 1), considerable differences between humans and rodents are obvious [5]. These differences limit the significance and validity of rodent models in the preclinical evaluation of some therapeutics, especially those with species-specific effects [6]. Therefore, established non-human primate models in macaques can be clearly favoured as preclinical models of CIA $[7,8]$. However, despite their advantages, the limitations of these monkey models, such as a low incidence of the disease, an acute but not chronic type of inflammation, as well as the large body size of the animals, hamper their usability in preclinical studies.

Aiming to develop a novel non-human primate model of CIA that overcomes the limitations of the pre-existing macaque models, Vierboom and colleagues investigated the common marmoset, a small-sized primate that has already been studied in models of experimental autoimmune encephalomyelitis. Common marmosets immunized with either bovine or chicken CII developed autoimmune arthritis with very high incidence (21 out of the 22 experimental animals). Half of the animals developed acute transient arthritis, while the other half was characterized by chronic arthritis. Analysis of T cells and anti-CII antibodies suggests that both cellular and humoral immune responses are involved in the development of the disease. Due to the lack of suitable detection reagents, C-reactive protein as a biomarker of inflammation could not be determined. However, excreted lysylpyridinolines as biomarkers of bone erosion were observed in animals with severe disease. 
CIA is induced with heterologous CII in mice, rats and monkeys, with the exception of that induced in DA rat with rat CII [9]. Unlike autologous CII, heterologous CII can only occasionally induce autoreactive $\mathrm{T}$ cells in some animal strains. Although both heteroreactive and autoreactive $T$ cells can help $B$ cells to produce autoantibodies to $\mathrm{CII}$ and subsequently induce arthritis, the involvement of autoreactive $\mathrm{T}$ cells makes a significant difference in the pathogenesis of CIA [10] and the latter cell type should be characterized in a new model of CIA. The authors provided evidence of the presence of heteroreactive $\mathrm{T}$ cells in marmosets with severe disease, but clear proof of the presence of autoreactive T cells is missing. Together with identification of the corresponding immunogenic $\mathrm{T}$ cell epitopes, more detailed characterization will significantly increase the impact of this model.

Compared to the macaque models, the marmoset model of CIA shows several advantages. First of all, common marmosets are smaller and reach their adult age earlier than macaques, which saves capacity in animal facilities and reduces the amounts of tested substances in experiments. Second, the incidence of CIA in common marmosets is higher $(>95 \%)$ than that in macaques (40 to $60 \%$ in rhesus monkey). Both common marmosets and macaques are natural outbreds. The surprisingly high incidence observed in common marmosets, which might be due to a limited variability in their major histocompatibility complex loci [11], allows the evaluation of the efficacy of therapeutics using a small number of animals. Finally, in contrast to macaques, half of the common marmosets developed chronic arthritis. This chronic arthritis resembles strongly the pathology in humans and provides a wide time window for clinical treatment studies.

It is also interesting to note that marmosets are born as bone marrow chimeric twins [12], which are immunologically highly comparable. This unique nature allows a paired experimental setting, with one sibling treated with therapeutics and the other treated with placebo, which may be a valuable tool in preclinical studies.

Aside from the advantages of using marmosets, a main drawback of this model should be considered. The evolutionary distance between marmosets and humans is significantly larger than that between macaques and humans (Figure 1). Compared to macaques, marmosets differ much more from humans with regard to their physiology and immunology. These physiological differences may limit their validity in safety and toxicity tests. Disparities between humans and marmosets in the immune system will decrease the cross-reactivity of human-specific therapeutics and, furthermore, increase the immunogenicity of such therapeutics, which may result in the formation of neutralizing antibodies.
In conclusion, experimental CIA in marmosets provides a novel and promising non-human primate model of RA for preclinical research. With regard to its experimental handling and practicability, as well as its evolutionary distance from humans, this model will help to fill the gap between pre-existing models based on rodents and macaques. Further studies will evaluate the significance and validity of this model and its suitability for preclinical studies.

Abbreviations

CIA, collagen-induced arthritis; CII, type II collagen; RA, rheumatoid arthritis.

Competing interests

The authors declare that they have no competing interests.

Published: 30 November 2010

\section{References}

1. Vierboom MPM, Breedveld E, Kondova I, Hart BA: Collagen-induced arthritis in common marmosets: a new nonhuman primate model for chronic arthritis. Arthritis Res Ther 2010, 12:R200.

2. Trentham DE, Townes AS, Kang AH: Autoimmunity to type II collagen an experimental model of arthritis. J Exp Med 1977, 146:857-868.

3. Cathcart ES, Hayes KC, Gonnerman WA, Lazzari AA, Franzblau C: Experimental arthritis in a nonhuman primate. I. Induction by bovine type II collagen. Lab Invest 1986, 54:26-31.

4. Courtenay JS, Dallman MJ, Dayan AD, Martin A, Mosedale B: Immunisation against heterologous type II collagen induces arthritis in mice. Nature 1980, 283:666-668

5. Mestas J, Hughes CC: Of mice and not men: differences between mouse and human immunology. J Immunol 2004, 172:2731-2738.

6. Chapman K, Pullen N, Graham M, Ragan I: Preclinical safety testing of monoclonal antibodies: the significance of species relevance. Nat Rev Drug Discov 2007, 6:120-126.

7. Uchiyama Y, Yorozu K, Hashizume M, Moriya Y, Mihara M: Tocilizumab, a humanized anti-interleukin- 6 receptor antibody, ameliorates joint swelling in established monkey collagen-induced arthritis. Biol Pharm Bull 2008, 31:1159-1163.

8. Vierboom MP, Zavodny PJ, Chou CC, Tagat JR, Pugliese-Sivo C, Strizki J, Steensma RW, McCombie SW, Celebi-Paul L, Remarque E, Jonker M, Narula SK, Hart B: Inhibition of the development of collagen-induced arthritis in rhesus monkeys by a small molecular weight antagonist of CCR5. Arthritis Rheum 2005, 52:627-636.

9. Holmdahl R, Vingsbo C, Malmstrom V, Jansson L, Holmdahl M: Chronicity of arthritis induced with homologous type II collagen (CII) in rats is associated with anti-CII B-cell activation. J Autoimmun 1994, 7:739-752.

10. Malmstrom V, Michaelsson E, Burkhardt H, Mattsson R, Vuorio E, Holmdahl R: Systemic versus cartilage-specific expression of a type II collagen-specific T-cell epitope determines the level of tolerance and susceptibility to arthritis. Proc Natl Acad Sci U S A 1996, 93:4480-4485.

11. Antunes SG, de Groot NG, Brok H, Doxiadis G, Menezes AA, Otting N, Bontrop RE: The common marmoset: a new world primate species with limited Mhc class II variability. Proc Natl Acad Sci U S A 1998, 95:11745-11750.

12. Benirschke $K$, Anderson JM, Brownhill LE: Marrow chimerism in marmosets. Science 1962, 138:513-515.

13. ClustalW2 [http://www.ebi.ac.uk/Tools/msa/clustalw2/]

doi:10.1186/ar3181

Cite this article as: Petersen F, Yu X: A novel preclinical model for

rheumatoid arthritis research. Arthritis Research \& Therapy 2010, 12:148. 